\title{
Die Rolle der Spitalapotheke in klinischen Studien
}

\author{
Vera R. Mitter ${ }^{\mathrm{a}}$, Isabelle Sommer ${ }^{\mathrm{b}}$, Hélène Schaller ${ }^{\mathrm{c}}$, Stefanie Deuster ${ }^{\mathrm{d}}$, Jeannette Goette \\ ${ }^{a}$ Apothekerin, PhD, Institut für Spitalpharmazie Inselspital, Universität Bern; b Apothekerin, PhD, Spitalpharmazie Centre Hospitalier Universitaire Vaudois \\ (CHUV), Lausanne; ${ }^{c}$ Apothekerin, FPH Spitalpharmazie, Institut für Spitalpharmazie Inselspital, Universität Bern; ${ }^{d}$ Apothekerin, PhD, FPH Spitalpharmazie,

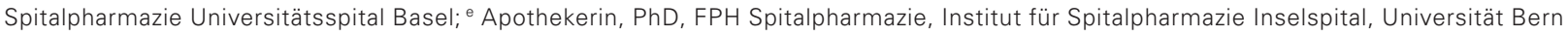 \\ Für die Arbeitsgruppe "Klinische Versuche» der GSASA (Schweizerischer Verein der Amts- und Spitalapotheker)
}

\section{Die Spitalapotheken der Kantons- und Universitätsspitäler bieten neben der Her- stellung von und Versorgung des Spitals mit Arzneimitteln auch wichtige Dienst- leistungen für klinische Studien an.}

Spitalapotheken kümmern sich im Rahmen von klinischen Studien um Prüfpräparate, sogenannte Investigational medicinal products (IMPs), und die Studienkoordinatoren sind die Spezialisten dafür. Sie arbeiten mit den Studienteams, den Clinical Trial Units (CTUs) und Swissmedic zusammen. An Universitäts-, Kantons- und Regionalspitälern werden vor allem Studien mit Pharmafirmen oder Non-Profit-Organisationen als Sponsoren («Sponsor-Studien») durchgeführt. An universitären Zentren gibt es auch von Prüfärzten (Principal Investigator) initiierte Studien (Investigator-Initiated Clinical Trials, IICTs).

\section{Rechtliche Grundlagen und regula- torische Anforderungen}

Good Clinical Practice (GCP) und Good Manufacturing Practice (GMP) sind für die Durchführung klinischer Studien verbindlich. Das schweizerische Humanforschungsgesetz (HFG, SR 810.30) verweist in Artikel 10 auf GCP und das Heilmittelgesetz (HMG, SR 812.21) in Artikel 7 auf GMP. Annex 13 der GMP regelt die speziellen Herstellungsanforderungen an IMPs. Art und Verwendung des IMP in der Studie entscheiden über die Kategorisierung gemäss Verordnung über klinische Versuche in der Humanforschung, Artikel 19 (KlinV, SR 810.305):

\section{Definition}

Wortlaut der Definition von Investigational medicinal products (IMPs) gemäss Good Clinical Practice (GCP):

«Die Darreichungsform eines wirksamen Bestandteils oder Placebos, die in einer klinischen Prüfung getestet oder als Referenz verwendet wird. Hierunter fällt auch ein bereits zugelassenes Produkt, wenn es in anderer Form (andere Darreichungsform oder Verpackung) als zugelassen verwendet oder bereitgestellt oder für ein nicht zugelassenes Anwendungsgebiet oder zum Erhalt weiterer Informationen über ein zugelassenes Anwendungsgebiet verwendet wird» [1].
- A: Das Arzneimittel wird gemäss Fachinformation in der zugelassenen Verpackung und Indikation oder einer Indikation in derselben Krankheitsgruppe oder bei einer selbstlimitierenden Krankheit (niedriger dosiert), oder gemäss internationaler Leitlinie eingesetzt.

- B: Das Arzneimittel ist in der Schweiz zugelassen, wird aber nicht gemäss A eingesetzt.

- C: Das Arzneimittel ist in der Schweiz nicht zugelassen.

Klinische Versuche der Kategorie B oder C müssen von Swissmedic bewilligt werden. Die Clinical Trial Application besteht aus 10 Abschnitten. Abschnitt 7 beinhaltet das Pharmaceutical Quality Dossier, welches die Herstellschritte inklusive verwendeter Ausgangsstoffe und Materialien, die In-Prozess-Kontrollen und den Freigabeprozess detailliert beschreibt. Es wird bei IICTs vorzugsweise von der Spitalapotheke als Herstellerin verfasst. In Abschnitt 8 wird die Herstellbewilligung der Spitalapotheke und in Abschnitt 9 die Angaben auf den Etiketten der IMPs eingefügt [2].

\section{Herstellbewilligung}

Für die «Herstellung» von IMPs bedarf es einer Herstellbewilligung von Swissmedic, die explizit die «Herstellung von Arzneimitteln für klinische Versuche» einschliesst.

Folgende Spitalapotheken verfügen über diese Herstellbewilligung (Stand Februar 2021):

- Universitätsspital Basel (USB)

- Ente Ospedaliero Cantonale Bellinzona (EOC)

- Inselspital Bern (Insel Gruppe AG)

- Hôpitaux Universitaires de Genève (HUG)

- Centre Hospitalier Universitaire Vaudois (CHUV)

- Kantonsapotheke Zürich (KAZ)

- Spitalapotheke Psychiatrische Universitätsklinik Zürich 


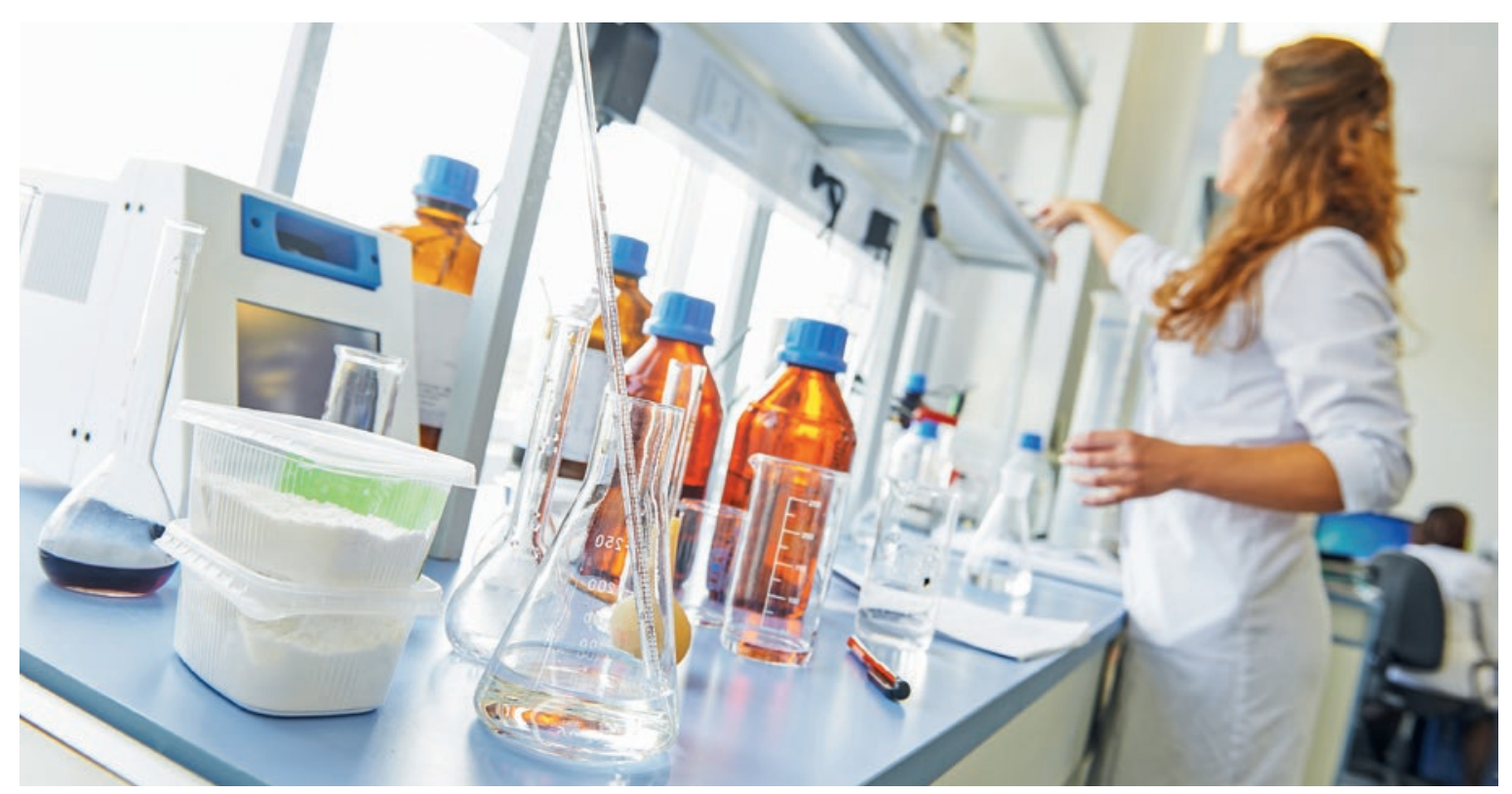

Herstellung und Zubereitung von Prüfpräparaten für Studien sind wichtige Aufgabenbereiche der Spitalapotheke.

Auf der Swissmedic-Homepage gibt es dazu eine Übersicht [3]. Die Herstellbewilligung beschränkt sich auf die darin genannten Arzneiformen. Nicht bewilligte Formen können in Lohnherstellung aus anderen Betrieben beschafft werden, weshalb der Weg über die Spitalapotheke auch da sinnvoll ist.

\section{Spitalapotheken kümmern sich bei klinischen} Studien um Prüfpräparate, sogenannte «Investigational medicinal products» (IMPs).

Wichtig ist die Abgrenzung zur «Zubereitung»: Wenn ein IMP gemäss Fachinformation oder Firmenvorschrift kurz vor der Anwendung am Patienten vorbereitet, d.h. rekonstituiert oder in einer Trägerlösung verdünnt und dem Studienteam übergeben wird, handelt es sich nicht um eine Herstellung und bedarf keiner Herstellbewilligung für klinische Studien.

\section{Sponsor-Studien}

In Sponsor-Studien liefert der Sponsor das IMP und ist verantwortlich für die Bewilligungen und Finanzierung der Studie. Die Spitalapotheke übernimmt die Lagerung und die patientenspezifische Rekonstitution in folgenden Situationen:

- Die IMPs onkologischer Studien werden meist state-of-the-art in der zentralen ZytostatikaAbteilung hergestellt.

- Die Infusions-/Injektionslösung muss verblindet abgegeben werden, kann aber nur unverblindet hergestellt werden.
- Der Sponsor verlangt die Rekonstitution in der Spitalpharmazie.

Ein Pharmacy Manual beschreibt das Vorgehen, auf dessen Grundlage die interne Herstellvorschrift erstellt wird oder die Stammdaten der Präparate im lokalen Informatikprogramm (z.B. CATO; computer-aided therapy for oncology) hinterlegt werden.

In Monitoringvisiten werden die vorgeschriebenen Abläufe und deren vollständige Dokumentation überprüft. Bei verblindeten Studien werden die Spitalapotheken von einem unverblindeten Monitor und das Zentrum von einem verblindeten Monitor geprüft. Die Close-out-Visite schliesst die Studie ab. Die Archivierung der geprüften Studiendokumente und die korrekte Entsorgung oder Rücksendung überflüssiger IMPs wird veranlasst.

\section{«Investigator-Initiated Clinical Trials» (IICTs)}

Die Aufgaben der Spitalapotheke im Rahmen von IICTs umfassen die Beratung, Machbarkeitsabklärung, Entwicklung von IMPs, Bearbeitung regulatorischer An-

Spitalapotheken mit einer Bewilligung für die Herstellung von IMPs verfügen häufig über Studienkoordinatoren.

forderungen und die Herstellung von ggf. unterschiedlichen IMPs für alle Behandlungsarme. Dazu gehören auch die Unterstützung in der Beschaffung von Ausgangsmaterial, Nachproduktion, Lagerung, Verteilung, 
Import oder Export sowie Entsorgung von IMPs. Fehlplanungen im Bereich der IMPs können ungeplante Nachproduktionen nach sich ziehen und rasch teuer werden. Ein vorzeitiger Studienabbruch wegen fehlender Finanzen wäre ethisch bedenklich [4].

Diese Aufgaben sind kostenintensiv, da sie stark reguliert sind. Zudem hängen sie von der Grösse, Komplexität und Dauer der Studie sowie den Anforderungen an das IMP ab. Im Rahmen der Verantwortlichkeitsabgrenzung werden Dienstleistungen und Vergütungen der Spitalapotheke vertraglich geregelt.

\section{Herstellung}

Die Stabilität des IMPs wird vor der Herstellung anhand der Ausgangsstoffe abgeschätzt. Bei eigenentwickelten IMPs können Stabilitätsuntersuchungen notwendig sein, die immer mit zusätzlichem Zeit- und Kostenaufwand verbunden sind.

Voraussetzung für die Herstellung der IMPs ist das Vorliegen der erforderlichen Bewilligungen und Herstellvorschriften. Herstellvorschriften werden vorgängig verfasst, validiert und implementiert. Sie beschreiben genau jeden Herstellschritt und notwendige In-Prozess-Kontrollen. Wird das IMP auf Vorrat hergestellt, braucht es zusätzlich Analyse- und Rückstellmuster. Am Anfang werden die Ausgangsstoffe und Packmaterialien beschafft, geprüft und freigegeben. Die effektive Produktion ist die hauptsächlich manuelle, teilweise halbautomatische Fertigung oder das Umverpacken (Abfüllen, Portionieren) sowie das (Um-)Etikettieren und Verblinden der IMPs. Jeder Herstellschritt wird dokumentiert. Nach Durchlaufen der Analyse und der Qualitätssicherung werden die hergestellten IMPs von der fachtechnisch verantwortlichen Person freigegeben. Die erste Patientenvisite (first patient, first visit) darf erst nach der Freigabe des IMPs stattfinden. Die Herstellräumlichkeiten, die Schulung des Personals sowie alle genannten Schritte der Herstellung,
In-Prozess-Kontrollen, Dokumentation und Freigabe sowie das Qualitätsmanagement müssen die Qualitätsvorgaben von GMP und Annex 13 einhalten. Ausgangsstoffe müssen ebenfalls den Qualitätsanforderungen nach GMP entsprechen.

\section{Logistik}

Die Anforderungen an Lagerung und Versand von IMPs wurden in den letzten Jahren durch die Richtlinien der Good Distribution Practice (GDP) zunehmend anspruchsvoll. Bei Transport und Versand müssen die geforderten Lagerbedingungen immer eingehalten werden. Spitalapotheken verfügen über qualifizierte Lagerorte für Raumtemperatur, Kühllagerung $\left(2-8^{\circ} \mathrm{C}\right)$ und Tiefkühler $\left(-20^{\circ} \mathrm{C}\right.$ und ggf. $\left.-80^{\circ} \mathrm{C}\right)$. Die Lagertemperaturen müssen mit jährlich kalibrierten Sensoren überwacht, dokumentiert und Abweichungen alarmiert werden.

\section{Rolle der Studienkoordinatoren}

Spitalapotheken mit einer Bewilligung für die Herstellung von IMPs verfügen häufig über Studienkoordinatoren. Sie bieten pharmazeutische Unterstützung und Beratung von der Planung bis zum Close-out einer klinischen Studie.

\section{Literatur}

1 Deutsche Übersetzung der Arbeitsgruppe des Bundesverbandes der Pharmazeutischen Industrie und des Verbandes Forschender Arzneimittelhersteller. Harmonisierte ICH-Leitlinie, Leitlinie zur Guten Klinischen Praxis.

2 www.swissmedic.ch/swissmedic/de/home/humanarzneimittel/ clinical-trials/klinische-versuche-mit-arzneimitteln/gesuch-zurbewilligung-eines-klinischen-versuchs.html

3 www.swissmedic.ch/swissmedic/de/home/humanarzneimittel/ bewilligungen_zertifikate/betriebsbewilligungen/bewilligungsinhaber.html

4 Kasenda B, von Elm E, You J, Blumle A, Tomonaga Y, Saccilotto R, et al. Prevalence, characteristics and publication of discontinued randomized trials. JAMA. 2014;311:1045-51.
Dr. sc. nat. Jeannette Goette Institut für Spitalpharmazie Insel Gruppe AG

Freiburgstrasse 4 CH-3010 Bern jeannette.goette[at]insel.ch

\section{Das Wichtigste in Kürze}

- Die Spitalapotheke ist bereits frühzeitig in die Planung zu involvieren.

- Das Portionieren, Umpacken und Etikettieren zur Verblindung von zugelassenen Medikamenten für klinische Versuche gilt als Herstellung.

- Eine Bewilligung zur Herstellung von Arzneimitteln für klinische Versuche wird benötigt.

- Kosten und Aufwand für die Herstellung von Investigational medicinal products (IMPs) werden oft unterschätzt.

\section{L'essentiel en bref}

- La pharmacie hospitalière doit être mise en place à un stade précoce de la planification.

- Le conditionnement, le réemballage et l'étiquetage en double aveugle de médicaments approuvés pour des essais cliniques sont considérés comme de la fabrication.

- Une autorisation de fabrication de médicaments pour des essais cliniques est nécessaire.

- Les coûts et l'investissement liés à la fabrication des Investigational medicinal products (IMPs) sont souvent sous-estimés. 